\title{
Analysis and Characterization of Power System Nonlinear Oscillations Us- ing Hilbert Spectral Analysis
}

\author{
A.R. Messina ${ }^{*}, 1$, M.A. Andrade ${ }^{1}$, J.H. Hernández ${ }^{1}$ and R. Betancourt ${ }^{2}$ \\ ${ }^{1}$ Graduate Program in Electrical Engineering, Cinvestav, Mexico \\ ${ }^{2}$ Faculty of Electromechanical Engineering, Universidad de Colima, Mexico
}

\begin{abstract}
In this contribution, a systematic approach to analyze and characterize the temporal evolution of nonlinear, time-varying processes in power systems is developed. The method combines the Hilbert-Huang transform and concepts from vibrating systems theory and can be used to approximate the dynamic behavior of quasi-stationary oscillations. Using the notion of empirical mode decomposition, data from transient stability simulations is transformed into a series of mono-component signals. A new analytical framework for characterizing and modeling the nonlinear temporal evolution of the oscillations is presented and techniques for identifying the modal content of the most dominant motion components are developed. Analytical expressions are then obtained that provide approximate solutions to the instantaneous frequency and amplitude of the oscillation, and a physical interpretation of the model is provided. The efficiency of this technique for capturing the temporal evolution of the modal content of data from transient stability simulations is assessed. Results determined from the low-order models are found to be in qualitative agreement with detailed system simulations.
\end{abstract}

\section{INTRODUCTION}

The analysis and characterization of nonlinear, nonstationary inter-area oscillations has attracted significant attention in recent years. Transient oscillations may result from the loss of major transmission and generation resources and be complicated by control actions, switching events and changes in system topology and operating conditions [1-3]. Experience with the analysis of wide-area electrical disturbances shows that many transient oscillations may manifest highly complex phenomena, including nonlinear and nonstationary behavior $[3,4]$. Understanding and characterizing the dynamics of these oscillations is a major research challenge in power systems dynamics. Recent studies suggest that in the analysis of complex nonlinear oscillations, such as those resulting from major disturbances or sequential faults, modal interaction is particularly difficult to characterize because of the large number of potential modes involved in the interaction and the time scales in which they interact $[1,2]$.

An accurate model of transient processes must capture dominant temporal features of the observed system dynamics such as abrupt changes in modal content and to relate these features to specific aspects of interest. This is particularly true in the development of wide-area measuring and control systems in which the specific impact of devices or events on system dynamic behavior must be properly characterized [1, 4].

A number of methods for the analysis and characterization of nonlinear, time-varying oscillatory processes have been recently proposed that have the ability to detect large and abrupt changes in system performance. Among these approaches, time-frequency transformations hold consider-

*Address correspondence to this author at the Graduate Program in Electrical Engineering, Cinvestav, Mexico; E-mail: aroman @gdl.cinvestav.mx able promise for the analysis of temporal behavior of critical system modes.

A novel application of the Hilbert Huang method was recently reported by Messina et al. [4] who applied the technique to characterize inter-area oscillations from measured data. This approach is capable of explaining the nonlinear nature of the observed oscillations and permits tracking the temporal evolution of dominant modes.

The present work extends earlier efforts made in using Hilbert analysis [4-6] by developing a systematic approach to analyze and characterize the temporal evolution of nonlinear, time-varying processes in power systems. The technique builds on simple concepts from vibrating systems theory and is applicable to both, nonlinear and non-stationary processes. A new analytical framework for characterizing and modeling the nonlinear temporal evolution of the oscillations is presented and techniques for identifying the modal content of the most dominant motion components are developed. The goal is to be able to represent the key contents of the data in terms of the smallest number of intrinsic mode functions that have physical significance. A large case study is presented to illustrate the application of the procedures. Results suggest that a small number of dominant modes can recover the temporal evolution of complex oscillations.

\section{THE ANALYTIC SIGNAL}

Let $x(t)$ be a real-valued signal. The analytic signal, $z(t)$, is a complex function of time defined as [7]

$z(t)=x(t)+j \tilde{x}(t)=A(t) e^{j \theta(t)}$

where

$\tilde{x}(t)=\frac{1}{\pi} P \int_{-\infty}^{\infty} \frac{y(\tau)}{t-\tau} d \tau$ 
is the Hilbert transform of $x(t)$. The analytic signal represents a local time-varying wave in the complex plane $x, \tilde{x}$ [6]. The instantaneous amplitude of the analytic signal is defined as the length of the rotating phasor, $A(t)$, namely

$$
A(t)=\sqrt{x(t)^{2}+\tilde{x}(t)^{2}}
$$

with instantaneous phase given by

$\varphi(t)=\arctan \left(\frac{\tilde{x}(t)}{x(t)}\right)$

As time elapses, the phasor rotates in the $(x, \tilde{x})$ plane and its instantaneous angular speed defines the angular frequency of the complex signal given by $\omega(t)=\dot{\varphi}(t)$. Fig. (1) shows a conceptual representation of the analytic signal.

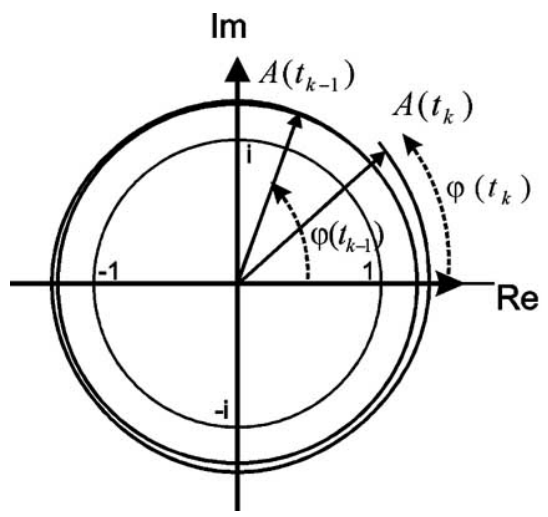

Fig. (1). Rotating phasor in the complex plane representing the analytic signal.

The extension to this approach to the analysis of multicomponent signals is possible using the concept of empirical mode decomposition (EMD) developed by Huang et al. [8]. Before presenting the method developed in this paper, a brief discussion of this procedure will be given.

\section{EMPIRICAL MODE DECOMPOSITION}

The concept of empirical mode decomposition was first introduced by Huang et al. [8]. The principle of this technique is to decompose a signal into a finite number of intrinsic mode functions (IMFs) that have the same numbers of zero crossings and extrema, and that are symmetric with respect to the local mean.

The IMFs are extracted serially, by fitting cubic splines to the envelopes of the maxima and minima of the function. At each step $k$ of the procedure, the highest frequency oscillation is removed. Following each iteration, a residue $r_{k}$ remains containing lower-frequency information. The process is repeated on the residue until only a trend remains; this results in a local decomposition of the data in which the intrinsic functions can be identified. 9]:

The EMD algorithm can be summarized as follows $[5,8$,
1. Initialize the procedure. Starting with the original signal $x(t)$, set the initial residue $r_{o}(t)=x(t)$, and $k=1$.

2. Extract the $k$ th IMF using a procedure called sifting.

3. Obtain an improved residue $r_{k}(t)$. Repeat step 2 with $k=k+1$ until the number of extrema in $r_{k}(t)$ is less than 2. This defines the trend of the signal.

The final result of this procedure is a decomposition of the form

$$
x(t)=\sum_{k=1}^{n} C_{k}(t)+r_{n}(t)
$$

where $n$ is the total number of IMFs, and $r_{n}$ is the nonoscillatory residual at the end of the sifting process.

The success of the technique is mainly due to the nature of the basis functions. By construction, the IMFs are almost orthogonal and form a complete basis (the sum of the individual IMFs equals the original signal). Each IMF corresponds to a different oscillatory time scale in the signal and is essentially a band-limited function.

Once the original signal is decomposed into a set of IMFs, the Hilbert transform is applied to each component to define the instantaneous characteristics of each component. Applying the Hilbert transform to each IMF, the original signal can be expressed as the real part of the complex expansion

$$
x(t)=\operatorname{Re} \sum_{k=1}^{n} z_{k}(t)=\operatorname{Re}\left(\sum_{k=1}^{n} A_{k}(\omega, t) e^{i \int \omega_{k}\left(t^{\prime}\right) d t^{\prime}}\right)
$$

where $z_{k}$ is the analytical signal associated with the $k$ th IMF, and $\omega_{k}(t)=\dot{\varphi}_{k}(t)$.

On the basis of the previous discussion, a technique is next proposed that enables the extraction of modal contents from simulated or measured data.

\section{MODAL PARAMETER IDENTIFICATION}

Representation of the observed oscillations by a loworder dynamic model, allows the efficient analysis of complex processes deriving from non-linear dynamical processes. Since the IMFs are nearly orthogonal to each other, each IMF is considered to be a harmonic oscillator of variable amplitude and frequency.

More precisely, consider a nonlinear single-degree-offreedom (sdof) system with nonlinear damping, modeled as

$$
\ddot{y}+2 \varsigma(t) \omega_{o}(t) \dot{y}+\omega_{o}^{2} y=0
$$

where $y(t)$ is the displacement of the mass, $\omega_{o}$ is the undamped natural frequency, and $\zeta$ is the dimensionless damping coefficient. Applying the Hilbert transform to (6) and combining real and imaginary parts yields a differential equation for the analytic signal [10]: 
$\ddot{z}+2 \varsigma(t) \omega_{o}(t) \dot{z}+\omega_{o}^{2} z=0$

Letting

$z(t)=A(t) e^{j \varphi(t)}=\Lambda e^{-\eta(t)} e^{i \varphi(t)}$

where $\eta(t)$ is an exponential factor characterizing the timedependent decay of the solution [11], it can readily be proved that

$\dot{z}(t)=z(t)[j \omega(t)-\dot{\eta}(t)]$

$\ddot{z}(t)=z(t)\left[i \dot{\omega}(t)-\omega(t)^{2}-2 i \omega(t) \dot{\eta}(t)-\ddot{\eta}(t)+\dot{\eta}(t)^{2}\right]$

and

$\frac{\dot{A}(t)}{A(t)}=-\dot{\eta}(t)$

$\frac{\ddot{A}(t)}{A(t)}=-\dot{\eta}(t)+\dot{\eta}(t)^{2}$

Substituting (10) into (9) and separating out the real and imaginary parts gives

$2 \zeta \omega_{o}(t)=2 \dot{\eta}(t)-\frac{\dot{\omega}(t)}{\omega(t)}$

$\omega_{o}(t)^{2}=\omega(t)^{2}+\dot{\eta}(t)-\dot{\eta}(t)^{2}-2 \zeta \omega_{o}(t) \dot{\eta}(t)$

Having determined the instantaneous amplitudes and frequencies (phases) from (2),(3), the damping and natural frequency of the nonlinear sdof systems can be obtained using (9). Fig. (2) shows a conceptual representation of the identification procedures applied to general data.

It is also of interest to determine the instantaneous energy of each oscillator. Using (8) the instantaneous energy of the sdof oscillator can be approximated in terms of the instantaneous amplitude and phase by

$e_{k}(t) \approx \Lambda_{k}{ }^{2} e^{-2 \eta_{k}(t)} \omega_{k}(t)^{2}$

Equations (11) and (12) completely characterize the modal behavior of the time series and the accuracy of the approximation can be assessed by comparing the contribution of successive coefficients.

While this procedure accurately represents the time evolution of the transient process, a global model is also needed that identifies dominant temporal features, and enables characterization of global attributes such as the instantaneous amplitude and frequency of the signal.

Based on the above model, a low-dimensional dynamic representation is proposed which allows us to approximate the dynamic behavior of quasi-oscillatory processes by a combination of a selected subset of the IMFs.

\section{QUASI-HARMONIC BEHAVIOR}

Low-dimensional models offer a compact description of the system dynamics, and they are potentially useful in analyzing, simulating and characterizing complex oscillations. In this section, a low-dimensional representation of temporal quantities is derived that enables to extract dominant modal information.
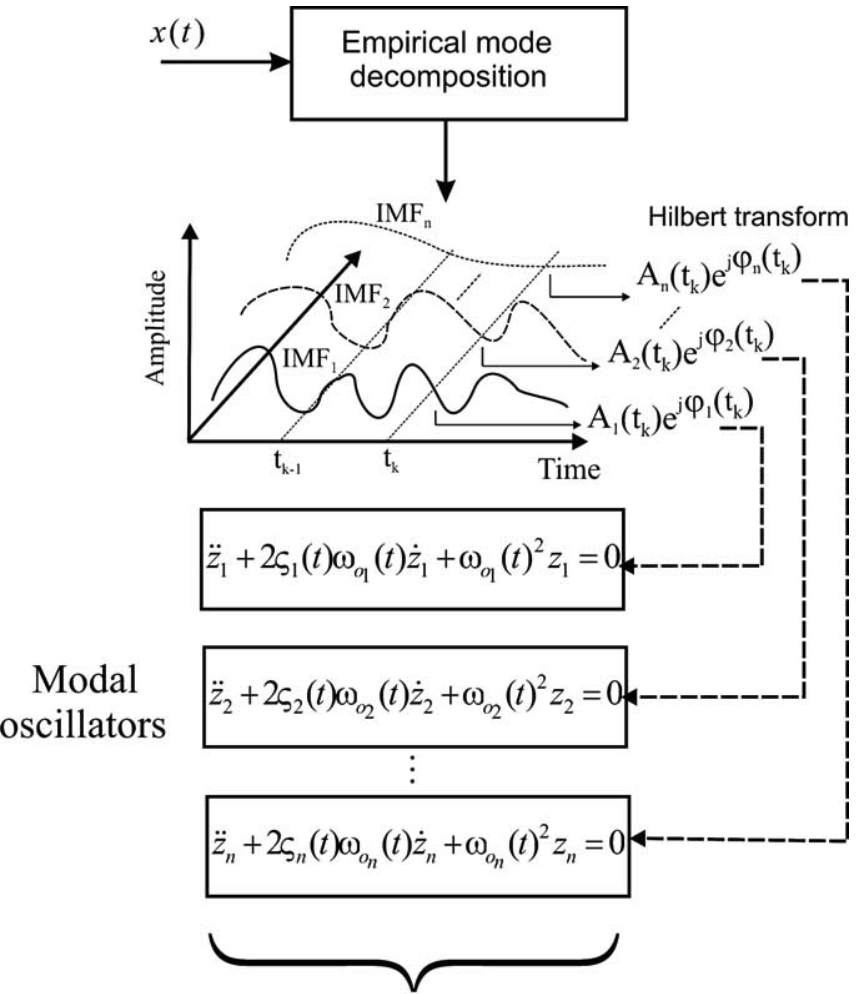

* Modal quantities

$\omega\left(t, A_{1}, \cdots A_{n}, \varphi_{1}, \cdots, \varphi_{n}\right)$

* Low-dimensional $A\left(t, \varphi_{1}, \cdots, \varphi_{n}\right)$ representations

* Instantaneous attributes

Fig. (2). Conceptual representation of the proposed technique.

Following Feldman [12, 13], assume that the observed signal, $x(t)$, can be approximated by family of $p$ oscillatory functions, with time-varying amplitudes $A_{j}$, and phases, $\varphi_{j}$ in the form $x(t)=\left[\sum_{j=1}^{p} A_{j}(t) \cos \left(\varphi_{j}(t)\right)\right.$ where the $\varphi_{j}(t)$ can be amplitude and/or frequency modulated.

Taking the Hilbert transform of this expression gives

$z(t)=\sum_{j=1}^{p} z_{j}(t)=\sum_{j=1}^{p} A_{j}(t) e^{i \varphi_{j}(t)}=A(t) e^{i \varphi(t)}$

and

$\dot{z}(t)=A(t) e^{i \varphi(t)} i \dot{\varphi}(t)+e^{i \varphi(t)} \dot{A}(t)$

where $p$ is the number of modal components, and $\omega(t), \varphi(t)=\int_{0}^{t} \omega(t) d t$ are the instantaneous frequency and phase to be solved for.

In terms of these variables we write the amplitude and frequency of the composite oscillation as

$$
A(\omega, t)=\sqrt{\sum_{j=1}^{p} A_{j}(t)^{2}+\sum_{k}^{p} \sum_{l \neq k}^{p} 2 A_{k} A_{l} \cos \left(\varphi_{k l}(t)\right)}
$$

and 


$$
\omega(A, t)=\operatorname{Im} \frac{\sum_{j=1}^{n}\left[A_{j} e^{i \varphi_{j}(t)} i \omega_{j}(t)+e^{i \varphi_{j}(t)} \dot{A}_{j}(t)\right]}{\sum_{j=1}^{p} A_{j} e^{i \varphi_{j}(t)}}
$$

where

$$
\varphi_{j}(t)=\int_{o}^{t} \omega_{j}(t) d t
$$

Based on these assumptions, we next derive a procedure for assessing the significance of modal components in system behavior. Our approach follows Feldman's treatment of nonlinear freely vibrating systems [12].

An interesting particular case arises when, for a physical system, the system response can be approximated by a few slowly varying functions or modal components.

Setting $\dot{A}_{j}(t) \approx 0$ in (16), and using the above relations one obtains,

$$
\omega(t)=\omega_{a v}+\frac{1}{2}\left[\frac{A_{2}(t)^{2}-A_{1}(t)^{2}}{A^{2}(t)}\right]\left(\omega_{2}(t)-\omega_{1}(t)\right)
$$

for $p=2$, where $\omega_{1}=\dot{\varphi}_{1}, \omega_{2}=\dot{\varphi}_{2}$,

$\omega_{a v}=0.5\left(\omega_{1}(t)+\omega_{2}(t)\right)$, and

$$
A(t)^{2}=A_{1}(t)^{2}+A_{2}(t)^{2}+2 A_{1}(t) A_{2}(t) \cos \varphi_{21}(t)
$$

where $\varphi_{21}(t)=\varphi_{2}(t)-\varphi_{1}(t)$.

Equation (17) defines a nonlinear and nonstationary frequency (amplitude)- modulated signal. Slightly more complicated expressions can be derived for the case of $n$ modal components and are not included here.

By systematically including third and higher order interactions, the quality of the approximation can be improved up to the desired order of accuracy. In the limit, when $n=p$, the signal can be fully reconstructed using (5).

In implementing the method, the instantaneous phases are obtained by unwrapping the phase angles $\varphi_{j}(t)$; the instantaneous frequencies are then computed using a thirdorder differential filter [14].

The high degree of accuracy offered by the proposed technique is next demonstrated by application to data from transient stability simulations in power system stability studies.

\section{APPLICATION}

Data from transient stability simulations of a large model of the Mexican interconnected system is used to examine characteristics of quasi-stationary processes. Reference [5] provides specific details of the study system and the contingencies under consideration.

Two contingency scenarios are selected for study:

Case A. Outage of Laguna Verde (LGV) unit \#1. This case assumes the outage without fault of unit \# 1 (650
MW) of the LGV nuclear power station, one of the two largest units in the system.

Case B. Simultaneous line outage without fault of one circuit of the $400 \mathrm{kV}$ line from MMT to JUI and the $400 \mathrm{kV}$ line from Temascal (TMD) to PBD on the $400 \mathrm{kV}$ network of the southeastern system.

These are the same contingencies used by the authors in previous studies [5].

Detailed transient stability simulations for the above contingencies were performed to generate the datasets. From these simulations, the real power signal of the $400 \mathrm{kV}$ transmission line from QRO to SLM (Case A) on the western system, and the $400 \mathrm{kV}$ transmission line from TMD to PBD on the southeastern part of the system (Case B) were selected for study. The time series are presented in Fig. (3a); the corresponding power spectra are shown in Fig. (3b).
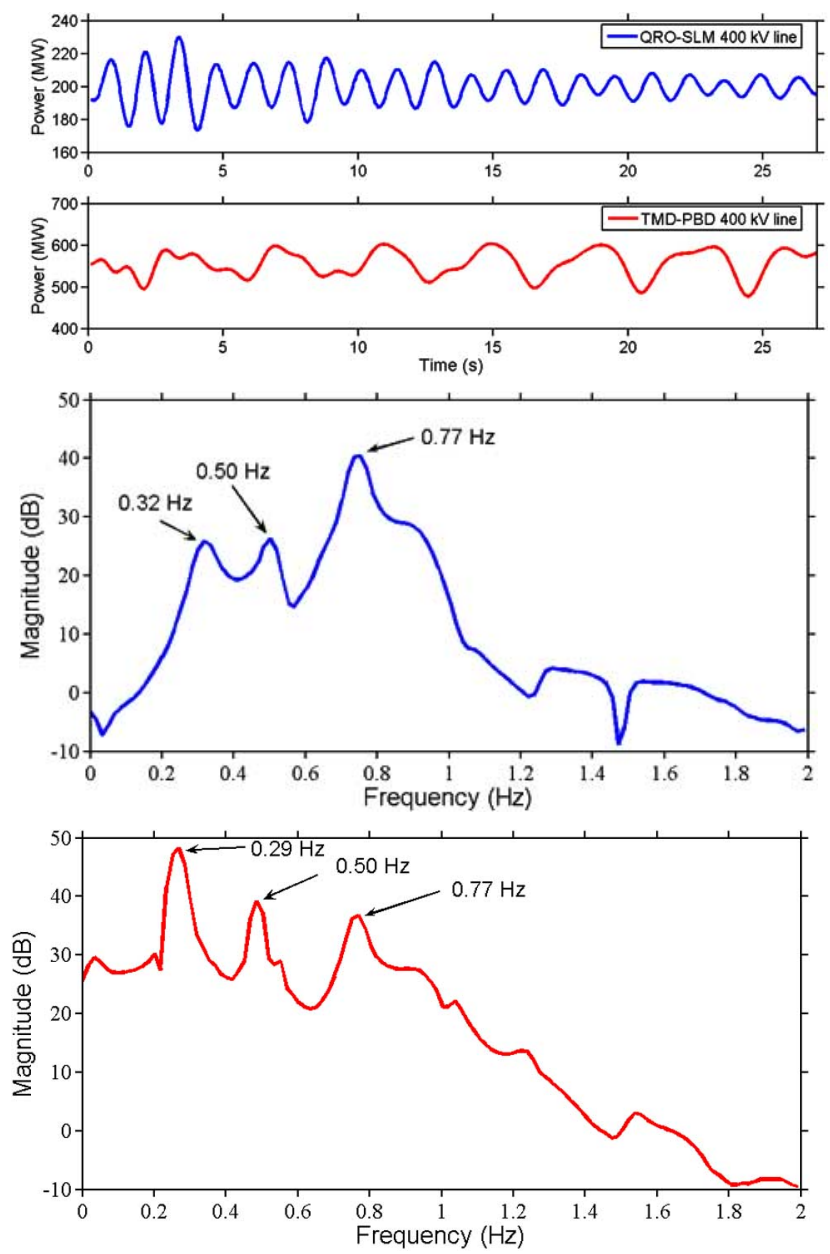

Fig. (3). Datasets. Top: Stability swing curves. Bottom: Fourier spectra.

Case A is an example of quasi-harmonic behavior whilst Case B exhibits a complex time-evolving frequency composition due to the control action of major system devices.

In the present simulations a time step of $0.016 \mathrm{~s}$ is used. Each of the above data sets consists of 2373 data points, corresponding to 30 s of data. 
For case A, the Fourier spectrum shows the presence of a dominant mode at about $0.77 \mathrm{~Hz}$. Two other participations are also noted at about $0.32 \mathrm{~Hz}$ and $0.50 \mathrm{~Hz}$. The analysis of case $\mathrm{B}$, on the other hand, reveals three dominant modes at about $0.29 \mathrm{~Hz}, 0.50 \mathrm{~Hz}$, and $0.77 \mathrm{~Hz}$.

\section{Analysis of Case A}

Following the approach in [5], the real power signal was decomposed into two IMFs and a trend. Fig. (4) shows the two dominant IMFs extracted using this procedure. For comparison, the approximate reconstructed signal obtained using the two dominant components (IMF1+IMF2) is also shown. The notable feature of both IMFs is periodicity with a frequency approximately equal to the frequencies in the Fourier spectra. These results clearly show that the time evolution of the observed signal can be approximated by the reduced order model (17),(18).
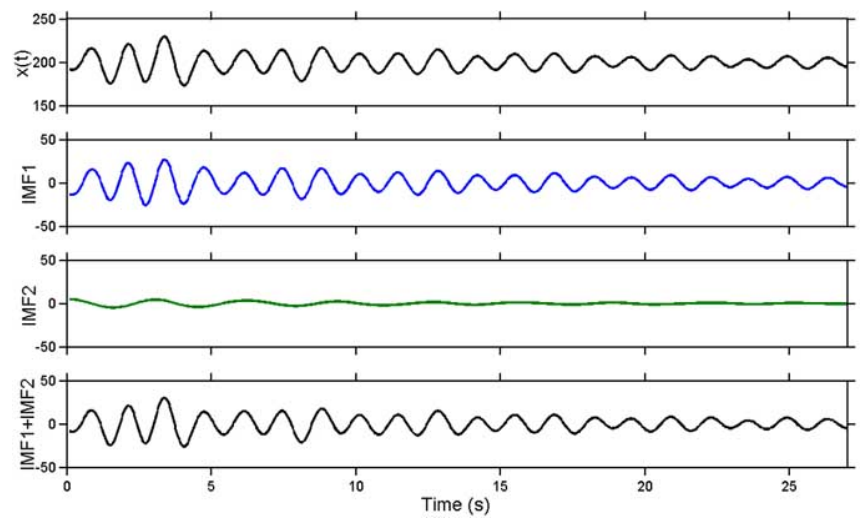

Fig. (4). Intrinsic mode functions. Case A.

Further insight into the nature of system behavior can be obtained from the study of instantaneous attributes in Fig. (5). The dominant IMF (IMF1) exhibits a nearly constant variation centered around $0.77 \mathrm{~Hz}$, whilst the second IMF is centered around $0.32 \mathrm{~Hz}$. In both cases the periodicity of the signals suggests that each components can be expressed as $I M F_{j}(t)=C_{j}(t)=A_{j}(t) \cos \varphi_{j}(t)$.

To gain fundamental insights into the physics of the oscillations we computed the instantaneous attributes of the dominant IMFs. Of particular interest are the amplitude $A_{j}(t)$, frequency $\omega_{j}(t)$, and phase $\varphi_{j}(t)$, which are plotted as a function of time for the two dominant IMFs, in Fig. (5).

The bottom panel of Fig. (5) shows the instantaneous phase together with the detrended instantaneous phases. This approach can be used to examine dynamic trends and phase relationships between key system signals.

A wealth of useful information can be obtained from Hilbert analysis of the observed behavior, as detailed in the following discussion. As observed in the lower panel of Fig. (5), the phase evolutions show increasing magnitudes. Simple theoretical considerations show that the slope of the instantaneous phases gives the frequency of oscillation; the greater the slope, the greater the frequency of the IMF. For the particular case under study, the temporal phases, $\varphi_{k}(t)$, $k=1, \cdots, 3$ exhibit a relatively well behaved linear trend over the entire period of study thus revealing the monocomponent nature of the simulated oscillations.

Interestingly, simulation results in Fig. (5) suggest that IMF1 and IMF2 exhibit some degree of phase coherence (locking) in which $\varphi_{1}-\varphi_{2}$ is approximately constant, i.e. IMF1 and IMF2 are in phase, near the end of the record. This fact has an important consequence in the interpretation of results as discussed below.

To further illustrate the potential usefulness of the method, we computed the instantaneous attributes of the signal. Fig. (6) shows the time evolution of the instantaneous amplitude of IMF1. Appropriate comparisons to both the second-order and third-order approximations instantaneous frequency and amplitude estimations, computed by using the analytical approximations in (17), (18) demonstrate that lowdimensional representations provide an accurate approximation to system behavior over the entire simulation.

Some interesting features of system dynamic behavior can be observed by examining the evolving frequency content and amplitude of the IMFs. As shown in Fig. (6), IMF1 is seen to provide a good approximation to system behavior especially for the final part of the record (15-30s). This coincides with the period of time in which the amplitude of IMF2 becomes negligible at about 20s (refer to the upper panel of Fig. (5)).

A key advantage of this approach is that the approximate analytical expressions filter the often partial or uninformative motion of the individual IMFs and isolate the most dominant motion components. This allows the periods of influence of each temporal mode to be clearly identified and characterized.

Fig. (7) shows the corresponding instantaneous frequencies. In each of the two panels of Fig. (7), the natural frequency of the fictitious oscillators determined using (11) and the mean instantaneous frequency (MIF) defined as the weighted average of the instantaneous frequencies

$$
\langle\omega\rangle_{t}=\frac{\sum_{k=1}^{n} a_{k}(t)^{2} \omega_{k}(t)}{\sum_{k=1}^{n} a_{k}(t)^{2}}
$$

were also plotted to facilitate physical interpretation.

As expected from previous considerations, a second order approximation to the instantaneous frequency accurately replicates system behavior. The physical interpretation of the IMFs is clear; the frequency of the first IMF coincides with the natural frequency, $\omega_{o}$, of the equivalent oscillator. Similar conclusions hold for other IMFs. We further observe that, as time evolves, the frequency of IMF1 approaches that of the second - and third-order approximations to the instantaneous frequency, $\omega(t)$, in agreement with previous findings.

These results demonstrate that the observed oscillations behave as a nonlinear sdof system in the frequency range of interest. As stated in our general conclusions, the use of a low-dimensional representation greatly simplifies the analy- 
sis of the observed time series and allows us to concentrate on the dominant modal behavior.
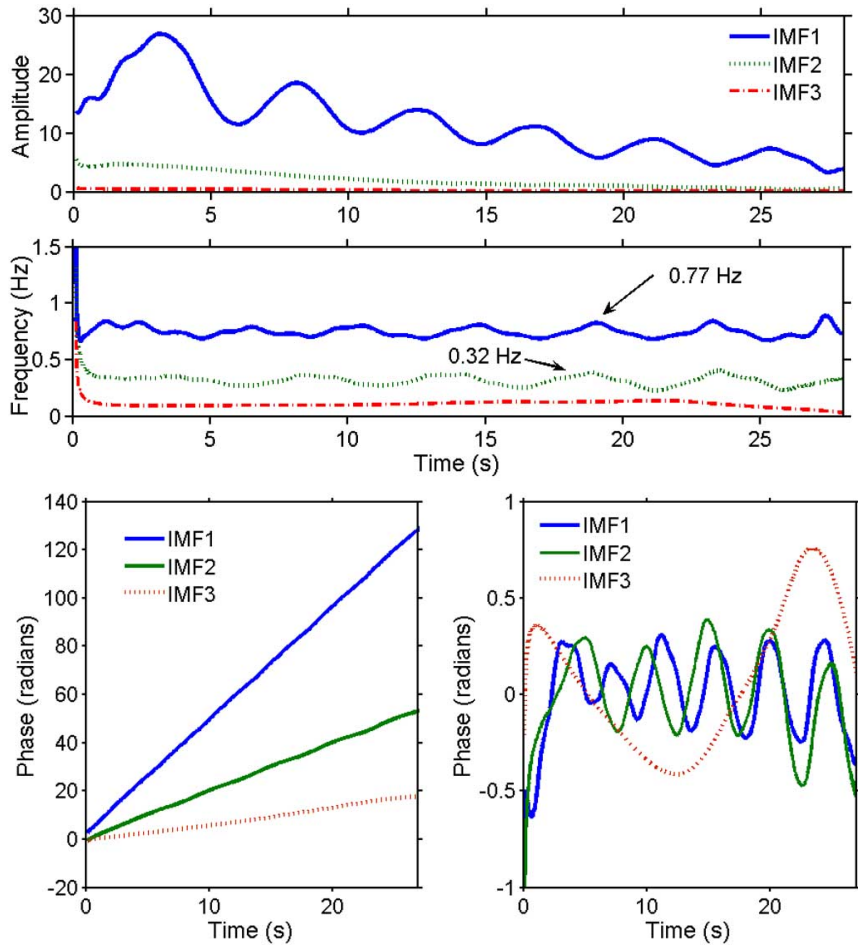

Fig. (5). Instantaneous attributes. Top: Amplitude and frequency. Bottom: Phase and detrended phase.

The quality of the model's prediction is indicative of the soundness of the model.

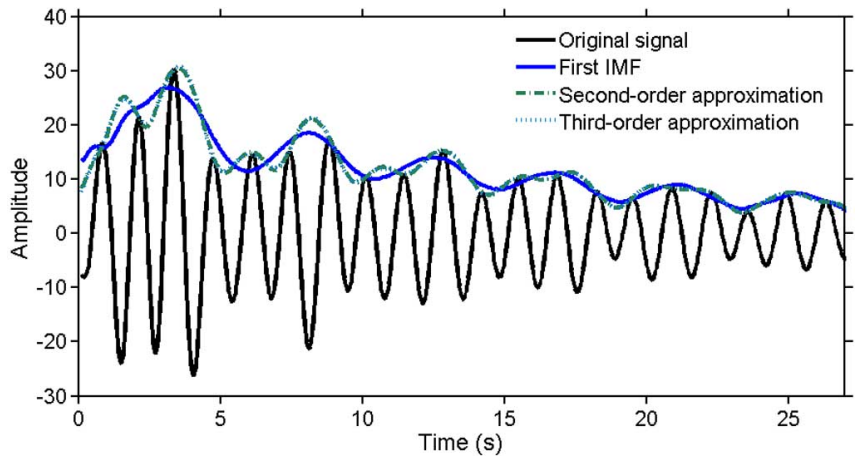

Fig. (6). Instantaneous amplitude.

\section{Analysis of Case B}

The analysis for this data set reveals a more complicated dynamics than in the previous case. Fig. (8) shows the dominant IMFs from EMD analysis. Note the strong nonlinear trend in the signal, $x(t)$. For comparison, the original signal is also plotted along with the reconstructed signal using the first two IMFs. (IMF1+IMF2); the remaining two modes show uninteresting behavior and are not studied here.

Upon visual inspection of the plots, the signal can be divided into two parts with different time scales, each exhibiting a quasi-stationary behavior. As observed in Fig. (8), IMF2 captures the dominant temporal evolution of the data for time window $2(0-12 s)$, while IMF1 is seen to capture system dynamics for time window 1 (12-25s).
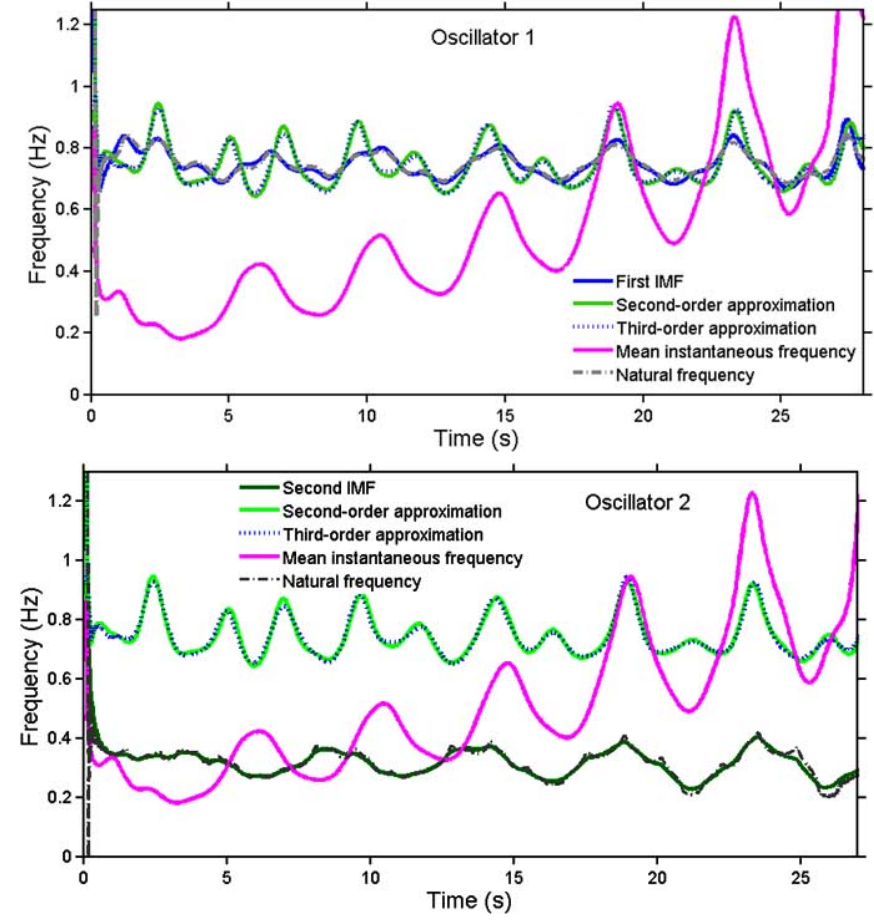

Fig. (7). Instantaneous frequency. Top: Oscillator 1. Bottom: Oscillator 2 .

Also noticeable in Fig. (8) is the presence of small riding waves which account for higher frequencies in the signal. Using this analysis, the exact arrival of the temporal modes can be identified which can be of importance to trigger control actions. Comparison of the time evolution of the original signal, $x(t)$, with the time evolution of the reconstructed signal (lower plot) shows that the EMD decomposition accurately reproduces the dynamic behavior for the entire observation period. The analysis also suggests that a lowdimensional representation enables accurate tracking of transient characteristics.
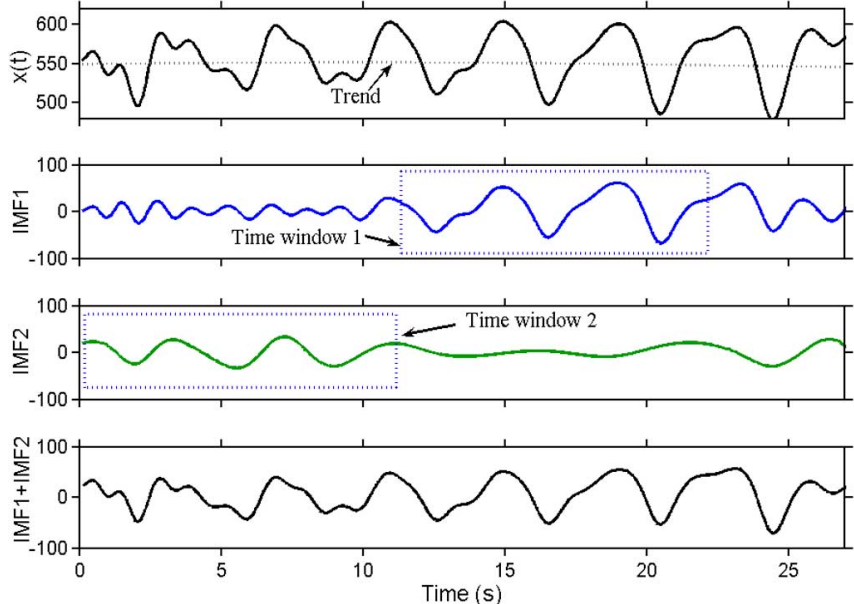

Fig. (8). Intrinsic mode functions. Case B.

On this basis, a detailed study of the ability of the proposed technique to extract the temporal behavior of the dominant modes was performed. Plots of the instantaneous parameters for IMFs 1 through 3 are shown in Fig. (9). 
The top panel of Fig. (9) shows the instantaneous energy, calculated from (12). The middle panel shows the corresponding time-dependent amplitudes and frequencies of the IMFs. Finally, the bottom panel shows the unwrapped (and detrended) instantaneous phases.
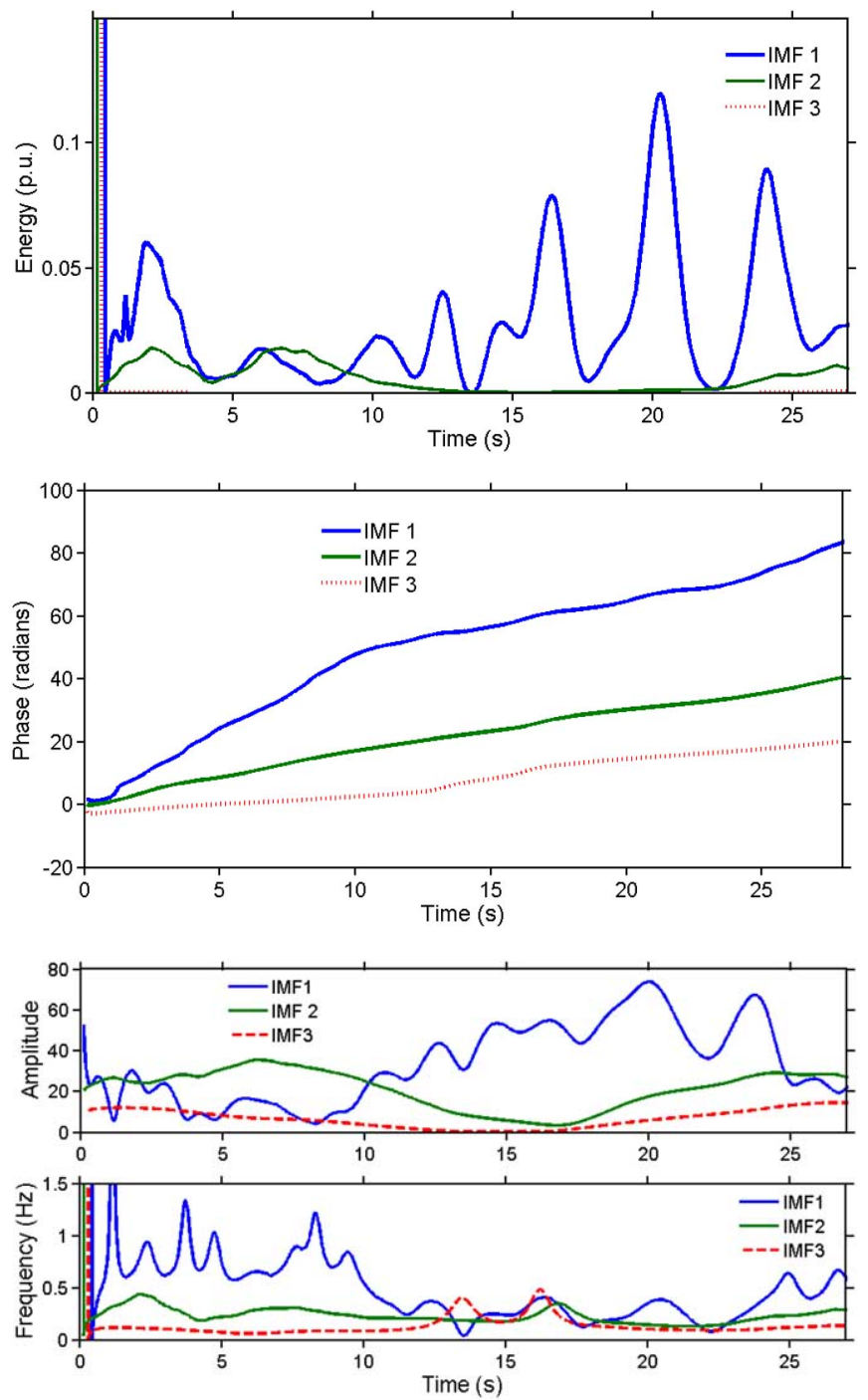

Fig. (9). Instantaneous attributes: Top: Energy, Middle: Phase. Bottom: Amplitude and frequency.

Hilbert analysis identifies significant temporal variability in the frequency content of IMF1. Both, amplitude and frequency modulation are observed. As would be expected from our previous analysis, IMF2 is seen to have a dominant contribution for the first part of the record, whilst IMF1 is seen to dominate the middle part of the oscillation. Clearly, a third-order approximation is needed to fully replicate system behavior especially for the initial $(0-10 \mathrm{~s})$ and the final part (20-30s) of the record. It is also interesting to observe that the instantaneous energy and amplitude of IMF1 indicate that the associated temporal mode becomes unstable near the end of the record in agreement with the behavior observed in Fig. (3). This is an important conclusion since the analysis suggests that this information might be used to trigger appropriate control actions.
Of special interest, the temporal phase of IMF1 shows abrupt slope changes at about $10 \mathrm{~s}$ and $25 \mathrm{~s}$ representing changes in the frequency content of the signal. This behavior coincides with variations in the frequency content of IMF1 (Bottom panel of Fig. (9)). In fact, our experience with the analysis of complex system oscillations indicates that routine estimation of temporal phase deviations provides critical information on the source mechanisms of oscillations and can be used to identify key relationships between system signals.

In an effort to verify the accuracy of the model, and to uncover the physical process underlying the oscillations, we computed the instantaneous attributes using a quasiharmonic approximation. Fig. (10) compares the instantaneous amplitudes and frequencies obtained using the first two IMFs, with those obtained from the second and third-order estimates in (15) and (16).
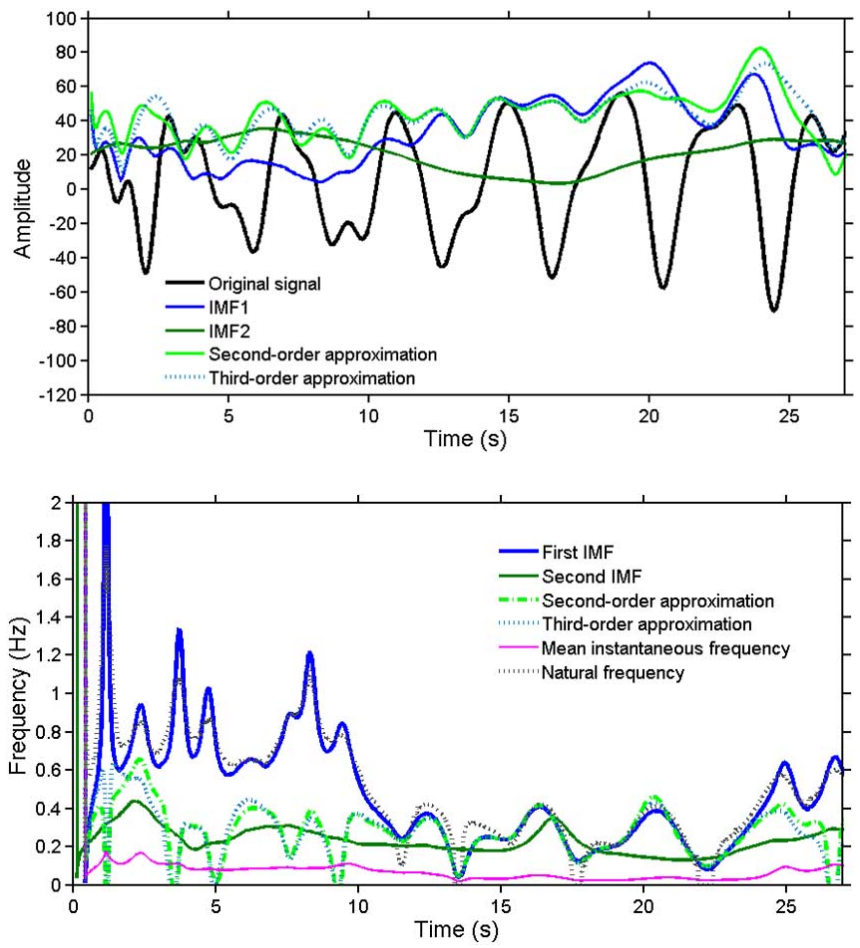

Fig. (10). Instantaneous amplitude and frequency. Top: Amplitude, Bottom: Frequency.

For direct comparison to the analytical estimates, the trend was removed from the signal, $x(t)$.

The results confirm that second and third-order approximations provide accurate characterization of transient behavior for the entire simulation period. It is interesting to note from the upper panel of Fig. (10) that, for time window 2 (012s), IMF2 behaves as the mid-amplitude around which the second and third-order approximations are centered. In contrast with this, for time window 1 (12-25s), IMF1 coincides with the low-dimensional instantaneous amplitude predictions indicating an essentially monocomponent behavior.

In turn, the frequency traces, depicted in Fig. (10), demonstrate significant variability. As is apparent from the lower panel of Fig. (10), the first IMF captures the dynamic behav- 
ior of time window 1, while IMF2 captures the dynamic behavior of the system for time window 2. For both periods, the analytical amplitude and frequency estimates provide an accurate representation of system behavior thus avoiding subjective interpretation of the modal content extracted from the behavior observed.

Finally, for the final part of the record, the approximate instantaneous frequency approaches the weighted average of that of IMFs 1 and 2. As noted in our previous discussion, the IMFs coincide with the natural frequency of the equivalent fictitious oscillators. This analysis provides a complete picture of the observed phenomena that is not easily available from the individual IMFs. Moreover, these results clearly indicate the efficacy of the procedure in extracting both local and average modal information. By judicious application of this technique, we can characterize complex oscillations in terms of critical, physically meaningful modes.

\section{CONCLUSIONS}

A new method of temporal representation of nonlinear, non-stationary processes in power systems has been presented. The analytical framework permits consideration of complex oscillatory processes and is applicable to both nonlinear and non-stationary processes.

The proposed technique was used to identify and extract temporal dominant features from relevant system variables. It is shown that observed oscillations can have a relatively simple dynamic behavior which only depends on a relatively small number of temporal modes. Such a model can be used for many purposes including reduction of system complexity, extraction of dominant features and wide-area monitoring and control. While the procedure has been illustrated for simulated data, the present method is quite general and extends readily to measured data. The use of this technique in conjunction with measured data from phasor measurement units appears promising for both, model validation and real time wide area post-disturbance analysis of system dynamics.

Techniques to improve the capacity of the proposed technique to extract rapid fluctuations from measured data as well as to characterize synchrony between multiple signals are under development.
Further work is also needed to improve the analytical approximations, especially for characterization of abrupt changes in system behavior.

\section{REFERENCES}

[1] J. F. Hauer, "Strategic issues in large-scale damping control", IEEE PES Summer Meeting, Symp on Inter-Area Oscillations. San Diego, CA. 1991.

[2] J. F. Hauer, and J. G. DeSteese, "A tutorial on detection and characterization of special behavior in large electric power system" $\mathrm{Pa}$ cific Northwest National Laboratory PNNL-14655 2004

[3] J. F. Hauer, and J. G. DeSteese, "A tutorial on detection and characterization of special behavior in large electric power systems" Pacific Northwest National Laboratory, Richland, WA, Rep. PNNL-14655, July 2004.

[4] A. R. Messina, V. Vittal, D. Ruiz-Vega, and G. Enriquez-Harper, "Interpretation and visualization of wide-area PMU measurements using Hilbert analysis", IEEE Trans. Pow. Syst., vol. 21, pp. 176361, November 2006.

[5] A. R. Messina, and V. Vittal, "Nonlinear, non-stationary analysis of inter-area oscillations via Hilbert spectral analysis", IEEE Trans. Pow. Syst., vol. 21, pp. 1234-41, August 2006.

[6] M. A. Andrade, A. R. Messina, C. A. Rivera, and D. Olguin, "Identification of instantaneous attributes of torsional shaft signals using the Hilbert transform", IEEE Trans. Pow. Syst., vol. 19, pp. 142229, August 2004.

[7] R. B. Randall, "Frequency Analysis", Ed. Bruel and Kjaer, 3er. Ed. 1987.

[8] N. E. Huang, Z. Shen, S. R. Long, M. C. Wu, H. H. Shih, Q. Zheng, N. C. Yen, C. C. Tung, and H. H. Liu, "The empirical mode decomposition and the Hilbert spectrum for nonlinear and nonstationary time series analysis", Proc. Royal Soc. Lond., vol. 454, pp. 903-95, 1998.

[9] P. Flandrin, G. Rilling, and P. Goncalves, "The empirical mode decomposition as a filter bank" IEEE Sign. Proc. Lett., vol. 11 (2), pp. 112-114, February 2004

[10] K. Worden, and G. R. Tomlinson, "Nonlinearity in structural dynamics, Detection, Identification, and Modelling", Institute of Physics Publishing, Bristol, Bristol, U.K: Institute of Physics, 2001, pp. 181.

[11] R. R. Zhang, L. VanDemark, J. Liang, and Y. Hu. "On estimating site damping with soil nonlinearity from earthquake recordings", Int. J. Nonl. Mech., vol. 39, pp. 1501-17, November 2004.

[12] M. Feldman, "Considering high harmonics for identification of non-linear systems by Hilbert transform", Mech. Syst. Sig. Proc., vol. 21, pp. 943-58, February 2007.

[13] M. Feldman, "Nonlinear free vibration identification via the Hilbert transform", J. Sound Vib., vol. 208, pp. 475-89, December 1997.

[14] P. D. Spanos, A. Giaralis, and N. P. Politis, "Time-frequency representation of earthquake accelrograms and inelastic structural response records using the adaptive chirplet decomposition and empirical mode decomposition", Soil Dyn. Earth Eng., vol. 27, pp. 675-89, July 2007 